\title{
BMJ Open Rehabilitation interventions in children and adults with infectious encephalitis: a systematic review protocol
}

\author{
Shanice Christie, ${ }^{1}$ Vincy Chan, ${ }^{2}$ Tatyana Mollayeva, ${ }^{3}$ Angela Colantonio ${ }^{3}$
}

To cite: Christie S, Chan V, Mollayeva T, et al. Rehabilitation interventions in children and adults with infectious encephalitis: a systematic review protocol. BMJ Open 2016;6:e010754. doi:10.1136/bmjopen-2015010754

- Prepublication history and additional material is available. To view please visit the journal (http://dx.doi.org/ 10.1136/bmjopen-2015010754).

Received 3 December 2015 Revised 21 January 2016 Accepted 1 March 2016

\section{CrossMark}

\footnotetext{
${ }^{1}$ Dalla Lana School of Public Health, University of Toronto, Toronto, Ontario, Canada ${ }^{2}$ Toronto Rehabilitation Institute, Toronto, Ontario, Canada

${ }^{3}$ Rehabilitation Sciences Institute, University of Toronto, Toronto, Ontario, Canada
}

Correspondence to Shanice Christie; shanice.christie@mail. utoronto.ca

\section{ABSTRACT}

Introduction: Many encephalitis survivors can benefit from rehabilitation. However, there is currently no comprehensive review describing rehabilitation intervention outcomes among children and adults with infectious encephalitis. This is a protocol for a systematic review that will summarise the current literature on outcomes following rehabilitative interventions among children and adults with infectious encephalitis. With a sufficient sample size, a sexstratified analysis of the findings will also be presented, as variability between male and female patients with neurological disorders, including encephalitis, regarding outcomes after rehabilitative interventions has been noted in the literature.

Methods and analysis: This review will systematically search MEDLINE, MEDLINE In-Process, EMBASE, Cochrane Central Register of Controlled Trials, and PsycINFO using the concepts 'encephalitis' and 'rehabilitation'. Grey literature will be searched using Grey Matters: A practical search tool for evidence-based medicine and the Google search engine. In addition, reference lists of eligible articles will be screened for any relevant studies. 2 reviewers will independently evaluate the retrieved studies based on predetermined eligibility criteria and perform a quality assessment on eligible studies.

Ethics and dissemination: The results from this review hold the potential to advance our knowledge on the value of rehabilitative interventions targeting children and adults with infectious encephalitis and any sex differences among patients with regard to rehabilitative intervention outcomes. The authors will publish findings from this review in a peer-reviewed scientific journal (electronic and in-print) and present the results at national and international conferences.

Trial registration number: CRD42015029217.

\section{INTRODUCTION}

Encephalitis is an inflammatory disease that affects the brain membrane due to various infectious agents (ie, bacteria and viruses) that cause acute infection to the brain parenchyma. These infectious agents can later impact the function of the central nervous system with severe neurological sequelae and

\section{Strengths and limitations of this study}

A strength of this review is meeting a need for knowledge on various rehabilitative interventions and outcomes in patients with infectious encephalitis, highlighting any sex differences observed for this population.

- The results of this review hold the potential to have an impact on policy and practice by providing relevant data to identify and describe current rehabilitative approaches for infectious encephalitis.

- Another strength of this review is its high sensitivity and specificity of search terms while a potential limitation may be the exclusion of non-English language studies and publication bias.

- There may be a limited number of high level of evidence studies due to challenges in designing interventions for patients with encephalitis in a rehabilitation setting.

- Significant heterogeneity in the population of interest, intervention setting, level of impairment and multifactorial outcome considerations is expected.

disorder. ${ }^{1}$ Viral aetiologies account for 32 $57 \%$ of infectious cases (ie, due to any infecting viral microorganisms) ${ }^{2}$ of encephalitis while other infectious, postinfectious and non-infectious aetiologies represent a smaller proportion. $^{3-6}$

Encephalitis occurs worldwide with some aetiologies having a global distribution (ie, herpes viruses) and others in specific geographical areas (ie, arbo viruses). ${ }^{4}$ Although it is a relatively rare disease, encephalitis is of a public health importance worldwide due to its high mortality and morbidity rates. ${ }^{7}$ In Europe, approximately 10000 12000 clinical cases of viral tick-borne encephalitis is reported annually. ${ }^{8}$ In Canada, incidence for encephalitis-related hospitalisations was estimated to be 24028 hospitalisations (5.2 per 100000 ) between 1994 and $2008,{ }^{5}$ while the USA had 238706 
encephalitis-related hospitalisations (7.3 per 100000$)$ from 2000 to $2010 .^{3}$ In addition, England, from 2005 to 2009, had an estimated rate of 4.32-8.66 cases per 100000 for encephalitis. ${ }^{9}$ In terms of mortality, certain aetiologies of encephalitis have mortality rates between $10 \%$ to $30 \%$, with various forms of encephalitis more severe than others. ${ }^{10}$ For example, the herpes simplex virus encephalitis, a common infectious type of encephalitis, has a mortality rate of up to $30 \%$ with specific antiviral treatment and $70-80 \%$ with no treatment. $^{10}$

Research on the costs of encephalitis has shown that it is considerable, despite relatively low rates of encephalitis-related hospitalisations (eg, 4.32-8.66 per 100000 within Canada, the USA and England). ${ }^{3}{ }^{5} 9$ According to Vora et $a l,{ }^{11}$ in 2010 , an estimated $\$ 2.0$ billion of total patient charges were attributed to encephalitis-associated hospitalisations in the USA. In England, the cost to the National Health Service based on hospital services utilised by patients with encephalitis was estimated to be US $\$ 60$ million per year. ${ }^{9}$

Encephalitis has been reported to affect all ages; however, it is more common among children, immunecompromised individuals and the elderly. ${ }^{12}{ }^{13}$ Morbidity following different types of encephalitis varies considerably. ${ }^{14}{ }^{15}$ Specifically, viral encephalitis has been associated with $20 \%$ of diagnosed patients having residual deficits. ${ }^{14}$ These deficits can include permanent neurological impairments, movement disorders, aphasia, behavioural abnormalities, amnestic syndromes, cognitive problems, and motor or sensory deficits. ${ }^{14}$ The recovery time from encephalitis and its related sequelae also varies. Although many patients may have a rapid and complete recovery within days to weeks, ${ }^{16}{ }^{17}$ a significant number of patients may experience incomplete recovery from encephalitis ${ }^{18} 19$ and experience sequelae. Such sequelae can be targeted and alleviated by rehabilitative interventions.

Despite the clinical and public health significance associated with encephalitis, outcomes following rehabilitative interventions for patients diagnosed with infectious encephalitis are sparsely documented. Previous studies examining outcomes of patients with encephalitis in rehabilitation have recognised the infrequent appearances of this population in rehabilitation programmes and the growing importance of admitting these patients to such programmes. ${ }^{14}$ 15 These infrequent appearances may be attributed to the finding that almost $50 \%$ of the encephalitis-related hospitalisations in Canada and $35 \%$ of cases from the USA have not been diagnosed as a specific encephalitis aetiology. ${ }^{3}{ }^{5}$ As a result, clinicians may continue to face the difficult task of selecting appropriate interventions that are likely to benefit patients with encephalitis. ${ }^{14}$

With respect to sex-specific outcomes after rehabilitative interventions, research on rehabilitation outcomes among patients with stroke has documented differences between males and females. ${ }^{20}{ }_{21}$ For example, differences in the risk of developing certain neurological disorder, ${ }^{22}$ their symptoms manifestation and severity, ${ }^{23}$ and how well males and females respond to intervention $^{2425}$ and seek healthcare ${ }^{26}$ have been documented in the literature, all which call for findings and any trends observed to be presented through a sex-stratified approach.

To the best of our knowledge, there is currently no systematic review on rehabilitation outcomes among patients with infectious encephalitis or sex-stratified evidence of sex differences regarding rehabilitation outcomes. Thus, the purpose of this systematic review protocol is to describe the methodology of a review that will summarise and identify evidence using a best evidence synthesis approach ${ }^{27}$ from all included studies examining the rehabilitative intervention outcomes among children ( $\leq 19$ years old) and adult patients $(>19 \text { years old })^{28}$ with a primary diagnosis of infectious encephalitis (ie, aetiology accounting for largest proportion of diagnosed known causes (23-42\%) for encephalitis ${ }^{3-6}$ while applying a sex-stratified approach, if feasible.

\section{METHODS AND ANALYSIS}

\section{Criteria for considering studies in the review}

The inclusion criteria for the title and abstract screen will consist of including studies reporting outcome data of rehabilitative interventions delivered to patients with a primary diagnosis of infectious encephalitis in an inpatient, outpatient or community rehabilitation setting. 'Rehabilitation' for this review will be defined using the WHO's definition of: 'any intervention that includes a process aimed at enabling patients to reach and maintain either their optimal physical, sensory, intellectual, psychological, or social functional levels by providing disabled patients with the tools they need to attain independence and self-determination'. ${ }^{29}$ Also for the first screen, this review will consider all published English language studies focused on human participants published before 1 June 2015 as well as all relevant experimental (ie, randomised controlled trials and pseudorandomised controlled trials), comparative (nonrandomised and observational, ie, concurrent or historical control, cohort, case-control, interrupted time series) and other observational (ie, case series, pretest/ post-test) studies. For this review, there is a preference to be inclusive during the first screen, and as such, no exclusionary criteria will be linked to the study design and targeted age group for studies looking at rehabilitation outcomes for encephalitis.

This review will apply the following exclusion criteria for the full-text screen: (1) theoretical articles or review of treatment approaches; (2) studies describing pharmacological-based interventions not focused on rehabilitation; (3) studies not providing predata/postdata of intervention of interest; and (4) studies presenting explicit patient data. 


\section{Search strategy}

This review will be reported in compliance with the Preferred Reporting Items for Systematic Reviews and Meta-Analyses (PRISMA) guidelines for systematic reviews. ${ }^{30}$ This review will systematically search the following electronic databases:

1. MEDLINE In-Process and Other Non-Indexed Citations and MEDLINE (from 1946),

2. EMBASE (from 1974),

3. PsycINFO (from 1805),

4. CINAHL (from 1981),

5. Cochrane Central Register of Controlled Trials (from 2005).

The search strategy for this protocol (see online supplementary additional file 1) was derived using relevant published reviews as guides ${ }^{31} 32$ and through consultation with an information specialist. Two concepts, (1) encephalitis and (2) rehabilitation, were chosen for this search strategy. The concept 'encephalitis' includes the proxy terms: 'encephalitis', 'encephaliti*', 'encephalopath*', 'Rasmussen* syndrome' and 'brain inflammation'. This was done to increase the likelihood of capturing studies that included patients with infectious encephalitis. The second concept, 'rehabilitation', includes a wide range of terms related to rehabilitative interventions (ie, 'physical', 'occupational', 'speechlanguage'). This was done in order to capture a variety of rehabilitation services used by patients diagnosed with infectious encephalitis. Additional proxy terms under the 'rehabilitation' concept that related to 'outcome' included 'length of stay', 'recovery of function', 'functional outcome', 'functional independence measure', 'functional recovery', 'discharge destination' and 'discharge status'. This was done to ensure that studies documenting rehabilitation outcomes of various interventions would be included.

In addition, grey literature will also be searched concurrently with the database search using the Google search engine and 'Grey Matters: a practical search tool for evidence-based medicine'. As advised by the consulted information specialist, the first 50 results retrieved by the Google search engine and 'Grey Matters' will be considered. The reference lists of eligible studies that passed the full-text screen will also be manually searched to ensure a comprehensive review. This review will organise retrieved articles with Microsoft Excel and save search results in the electronic reference management system EndNote (V.X7).

\section{Study selection}

With the assistance of two reviewers, a two-stage screening process for selecting eligible studies will be undertaken. First, using the inclusion criteria for the title and abstract screen, eligible studies will be identified and duplicates will be removed. In order for a study to pass the first screen, the record must have an English language version available that examines the rehabilitation of patients identified with having a primary diagnosis of infectious encephalitis in an inpatient, outpatient or community setting. The two reviewers will compare their records and come to a consensus before beginning the full-text screen. Any disagreements for the inclusion of studies will be referred to a third reviewer. Selected studies will then be considered for a full-text screen. During the full-text screen, two researchers will independently assess full-text studies using the inclusion and exclusion criteria. Once studies chosen for inclusion have been agreed on, reviewers will examine the reference lists of all selected studies to identify other potential eligible studies. Any disagreements for inclusion of studies will be referred to a third reviewer. A PRISMA flow diagram will be presented within the review with details of cause for rejection throughout the two database search screenings.

\section{Data extraction}

Two reviewers will independently extract data using a predefined table adapted from the Cochrane Collaboration handbook, which will be adjusted as necessary. This review will extract general data (authors, year of publication, country), participant characteristics (age and sex of the study population, type of infectious encephalitis, time since diagnoses of encephalitis), study characteristics (study design, intervention setting, purpose) and description of intervention and outcomes (table 1). Age of the study population will be categorised as children and adolescents ( $\leq 19$ years old) and adults (>19 years old).$^{28}$ We will review and openly report studies that did not provide sex-specific analysis. Both reviewers will compare collected data and any discrepancies will be referred to a third reviewer.

\begin{tabular}{|c|c|c|c|c|c|c|c|c|c|c|}
\hline \multicolumn{2}{|c|}{$\begin{array}{l}\text { General } \\
\text { characteristics }\end{array}$} & \multicolumn{4}{|c|}{ Participant characteristics } & \multicolumn{5}{|c|}{ Study characteristics } \\
\hline $\begin{array}{l}\text { Author } \\
\text { (year) }\end{array}$ & $\begin{array}{l}\text { Country } \\
\text { of study }\end{array}$ & $\begin{array}{l}\text { Age } \\
\text { (years) }\end{array}$ & $\begin{array}{l}\text { Sex } \\
\text { (M/ } \\
\text { F) }\end{array}$ & $\begin{array}{l}\text { Aetiology of } \\
\text { encephalitis }\end{array}$ & $\begin{array}{l}\text { Time } \\
\text { since } \\
\text { diagnosis } \\
\text { (months) }\end{array}$ & $\begin{array}{l}\text { Sample } \\
\text { size }\end{array}$ & $\begin{array}{l}\text { Study } \\
\text { design }\end{array}$ & $\begin{array}{l}\text { Intervention } \\
\text { setting }\end{array}$ & Intervention & Outcome \\
\hline
\end{tabular}


Table 2 Quality assessment of included studies

$\begin{array}{lllllll}\text { Study (first author) } & \text { Reporting (/10) } & \text { External validity (/3) } & \text { Bias (/7) } & \text { Confounders (/6) } & \text { Power (/5) } & \text { Total (/31) }\end{array}$

\section{Quality assessment of included studies}

The Downs and Black ${ }^{33}$ rating scale will be used for assessing the quality of the selected studies for this systematic review (table 2). Previously published peerreviewed systematic reviews focusing on rehabilitation evidence of moderate-to-severe acquired brain injuries have used the Downs and Black Tool for quality assessment. ${ }^{34} 35$ This instrument evaluates studies that use a non-experimental or uncontrolled design (prospective and retrospective controlled trials, single group interventions, retrospective and prospective cohort studies and cases studies). The scale consists of 27 questions, which are grouped into five subscales: reporting, external validity, bias and confounding, and overall power of the study with scores ranging from 0 to $34 .^{33}$ In the case of rating discrepancies, reviewers will come to a consensus or a third independent reviewer will evaluate the study to resolve any disagreements.

\section{Data synthesis}

This review will utilise a best evidence synthesis approach, integrating findings from studies with sufficient quality through tabulation and qualitative description. ${ }^{27}$ This approach considers that if included studies are not high in internal and external validity, then a careful analysis of the less well-designed studies can be performed in order to comprehend if there is adequate information to come to a meaningful conclusion. This review has established a consistent and clearly stated a priori inclusion and exclusion criteria, allowing us to capture all studies that meet broad standards in terms of both 'rehabilitation intervention' as well as 'encephalitis' categories. Thus, every study conducted that meets our inclusion criteria will be comprehensively reviewed and considered in our results and conclusions.

\section{Presenting and reporting the results}

This review will present results according to the PRISMA reporting guidance. ${ }^{30}$ The study selection process will be described in a flow chart with the inclusion and exclusion criteria described. Qualitative data will be presented in tables as narrative summaries describing characteristics of included studies, populations, description of interventions and measured rehabilitation outcomes (table 1).

\section{Ethics and dissemination}

As this review intends to use pre-existing published studies, ethical permissions will not be required. However, this review will follow the ethical and governance standards in the data management and presentation of results. The findings from this review will potentially be published in a peer-reviewed scientific journal (electronically and in print) and results will be presented at national and international conferences.

\section{Strengths and limitations}

The authors acknowledge there are inherent strengths and limitations of this review. A major strength of this review is meeting a need for knowledge of the rehabilitation interventions and outcomes of children and adults with a primary diagnosis of infectious encephalitis, while attempting to uncover any sex differences in rehabilitation outcomes between males and females. Another strength of this review is its high sensitivity and specificity of search terms that can provide a comprehensive literature search. In all, the results of this review hold the potential to have an impact on policy and practice by providing relevant findings to identify and describe current rehabilitative approaches for infectious encephalitis.

A potential limitation of this review is the exclusion of non-English language studies, as literature that focuses on rehabilitative interventions of patients with encephalitis aetiologies in a non-English language may not appear in the search. This may limit the comprehensiveness of this review. Also, due to the specificity of the targeted population for this review, publication bias may occur. Lastly, there may be a limited number of highlevel evidence studies due to challenges in designing standardised interventions for encephalitis in a rehabilitation setting.

\section{CONCLUSION}

To the best of our knowledge, this is the first protocol for a systematic review that will describe the rehabilitative intervention outcomes of patients with infectious encephalitis. This review will collate the literature to establish whether evidence suggests patients with infectious encephalitis show improvement using different interventions and may also provide valuable sex-specific data on the varying rehabilitation outcomes. The results of the review may also produce an important profile of patients with infectious encephalitis in a rehabilitation setting, which can contribute to improved planning and delivery of rehabilitation services by researchers, clinicians and policymakers.

Acknowledgements Jessica Babineau, an information specialist at the Toronto Rehabilitation Institute-University Health Network, assisted with developing the search strategy. The authors thank the reviewers of the manuscript for their constructive feedback. 
Contributors SC and VC conceptualised the study. SC formulated the design and drafted the manuscript. VC assisted with the development of the search terms and edited the manuscript. TM co-developed the design, supported SC in registering the protocol PROSPERO and critically reviewed the protocol. AC provided expertise at each level and also reviewed the protocol. All authors read and approved the final manuscript.

Funding $A C$ received support from the Canadian Institute of Health Research (CIHR) Chair in Gender Work and Health (\#CWG-126580). VC received support from the CIHR and Pediatric Oncology Group of Ontario for a Doctoral Research Award, Brain Canada and CIBC for a Brain Cancer Training Award and the Ontario Neurotrauma Foundation for the Jane Gillett Pediatric ABI Studentship. TM was supported by the postdoctoral fellowship from the RSI at the University of Toronto.

Competing interests None declared.

Provenance and peer review Not commissioned; externally peer reviewed.

Open Access This is an Open Access article distributed in accordance with the Creative Commons Attribution Non Commercial (CC BY-NC 4.0) license, which permits others to distribute, remix, adapt, build upon this work noncommercially, and license their derivative works on different terms, provided the original work is properly cited and the use is non-commercial. See: http:// creativecommons.org/licenses/by-nc/4.0/

\section{REFERENCES}

1. Meningitis and encephalitis fact sheet: National Institute of Neurological Disorders and Stroke (NINDS). http://www.ninds.nih. gov/disorders/encephalitis_meningitis/detail_encephalitis_meningitis. htm (accessed 15 Dec 2015).

2. Association of American Medical Colleges. Infectious Disease, Internal Medicine. 2015. https://www.aamc.org/cim/specialty/list/us/ 339608/infectious_disease_-internal_medicine.html (accessed 19 Nov 2015).

3. George BP, Schneider EB, Venkatesan A. Encephalitis hospitalization rates and inpatient mortality in the United States, 2000-2010. PLOS ONE 2014;9:e104169.

4. Khetsuriani N, Holman RC, Anderson LJ. Burden of encephalitis-associated hospitalizations in the United States, 19881997. Clin Infect Dis 2002;35:175-82.

5. Kulkarni MA, Lecocq AC, Artsob $\mathrm{H}$, et al. Epidemiology and aetiology of encephalitis in Canada, 1994-2008: a case for undiagnosed arboviral agents? Epidemiol Infect 2013;141:2243-55.

6. Granerod J, Ambrose HE, Davies NW, et al. Causes of encephalitis and differences in their clinical presentations in England: a multicentre, population-based prospective study. Lancet Infect Dis 2010;10:835-44.

7. Granerod J, Crowcroft NS. The epidemiology of acute encephalitis. Neuropsychol Rehabil 2007;17:406-28.

8. World Health Organization. Tick-borne Encephalitis. 2014. http:// www.who.int/immunization/topics/tick_encephalitis/en/ (accessed 22 Jun 2015).

9. Granerod J, Cousens S, Davies NW, et al. New estimates of incidence of encephalitis in England. Emerg Infect Dis 2013;19:1455.

10. The Encephalitis Society. Death from Encephalitis (acute stage). 1999. http://www.encephalitis.info/files/5913/4012/7875/FS063V1. pdf (accessed 11 Jan 2015).

11. Vora NM, Holman RC, Mehal JM, et al. Burden of encephalitis-associated hospitalizations in the United States, 19982010. Neurology 2014;82:443-51.
12. Gutierrez KM, Prober CG. Encephalitis-identifying the specific cause is key to effective management. Postgrad Med 1998;103: 123-43.

13. Kolski H, Ford-Jones EL, Richardson S, et al. Etiology of acute childhood encephalitis at The Hospital for Sick Children, Toronto, 1994-1995. Clin Infect Dis 1998;26:398-409.

14. Moorthi S, Schneider WN, Dombovy ML. Rehabilitation outcomes in encephalitis - a retrospective study 1990-1997. Brain Inj 1999;13:139-46.

15. Lowry PW. Abrovirus encephalitis in the United States and Asia. $J$ Lab Clin Med 1997;129:405-11.

16. Tong CYW, Potter FA, Pang KA. Severe encephalitis with rapid recovery, case report. Lancet 1997;349:470.

17. Zifroni A, Fried $M$, Shalmon $B$, et al. Severe encephalitis with rapid recovery. Lancet 1997;349:1328.

18. Fowler $\AA$, Forsman L, Eriksson M, et al. Tick-borne encephalitis carries a high risk of incomplete recovery in children. $J$ Pediatr 2013;163:555-60.

19. Fowler $\AA$, Stödberg T, Eriksson M, et al. Long-term outcomes of acute encephalitis in childhood. Pediatrics 2010;126:e828-35.

20. Zhao W, An Z, Hong Y, et al. Sex differences in long-term outcomes among acute ischemic stroke patients with diabetes in China. Biol Sex Differ 2015:6:1-10.

21. Mehndiratta P, Wasay M, Mehndiratta MM. Implications of female sex on stroke risk factors, care, outcome and rehabilitation: an Asian perspective. Cerebrovasc Dis 2015;39:302-8.

22. Peterson BL, Won S, Geddes RI, et al. Sex-related differences in effects of progesterone following neonatal hypoxic brain injury. Behav Brain Res 2015; 286:152-65.

23. Forsgren L, Nyström L. An incident case-referent study of epileptic seizures in adults. Epilepsy Res 1990;6:66-81.

24. Kappus RM, Ranadive SM, Yan $\mathrm{H}$, et al. Sex differences in autonomic function following maximal exercise. Biol Sex Differ 2015;6:1-8.

25. Zhou Y, Zhao M, Zhou C, et al. Sex differences in drug addiction and response to exercise intervention: from human to animal studies. Front Neuroendocrinol 2016;40:24-41.

26. Osika Friberg I, Krantz G, Määttä S, et al. Sex differences in health care consumption in Sweden: a register-based cross-sectional study. Scand J Public Health 2015. doi:10.1177/1403494815618843

27. Slavin RE. Best evidence synthesis: an intelligent alternative to meta-analysis. J Clin Epidemiol 1995;48:9-18.

28. World Health Organization. Adolescent Health. 2015. http://www. who.int/topics/adolescent_health/en/ (accessed 17 Nov 2015).

29. World Health Organization. Rehabilitation Health. 2015. http://www who.int/topics/rehabilitation/en/ (accessed 23 Jun 2015).

30. Moher D, Liberati A, Tetzlaff J, et al. Preferred reporting items for systematic reviews and meta-analyses: the PRISMA statement. Ann Intern Med 2009;151:264-9.

31. Khuu W, Chan V, Colantonio A. A systematic review protocol for measuring comorbidity in inpatient rehabilitation for non-traumatic brain injury. Syst Rev 2015;4:14.

32. Rohling ML, Faust ME, Beverly B, Effectiveness of cognitive rehabilitation following acquired brain injury: a meta-analytic re-examination of Cicerone et al.'s $(2000,2005)$ systematic reviews. Neuropsychology 2009;23:20-39.

33. Downs SH, Black N. The feasibility of creating a checklist for the assessment of the methodological quality both of randomised and non-randomised studies of health care interventions. J Epidemiol Community Health 1998;52:377-84.

34. Eng JJ, Teasell R, Miller WC, et al., SCIRE Research Team. Spinal cord injury rehabilitation evidence: methods of the SCIRE systematic review. Top Spinal Cord Inj Rehabil 2007;13:1-10.

35. Teasell R, Bayona N, Marshall S, et al. A systematic review of the rehabilitation of moderate to severe acquired brain injuries. Brain Inj 2007;21:107-12. 